DOI: 10.2478/awutm-2013-0016

侄

Analele Universităţii de Vest,

Timişoara

VERSITA

Seria Matematică - Informatică

LI, 2, (2013), 57- 71

\title{
Stability Analysis and its Impact on the Parameters Estimation for a Logistic Growth Model
}

Lavinia Cristina Moatăr-Moleriu

\begin{abstract}
This paper presents a stability analysis of a system of differential equations describing the evolution of T-cells populations. The analyzed system corresponds to a well-known four-compartmental model of the thymus which involves a logistic growth term. Unlike existing results on stability for this model which focus either only on the global population or on the two dominant double-positive and double-negative populations, the results presented in this paper provide sufficient conditions for asymptotic stability of all four populations, taken separately. The derived conditions involve parameters related to cells proliferation, death and transfer rates and are used as constraints in the least-square optimization procedure which provides estimates for all parameters of the model. The usage of the constraints derived from the stability analysis ensures that the estimated parameters lead to a mathematical model with an asymptotically stable fixed point.
\end{abstract}

AMS Subject Classification (2010). 93D20, 92B05, 68T20

Keywords. logistic growth, equilibrium points, stability, regression, parameter estimation, evolutionary algorithms. 


\section{Introduction}

Biological processes are usually complex, therefore identifying mathematical models able to reproduce the behaviour observed in experiments is a difficult task. Many biological processes involves one or several populations of cells which are involved in different kind of transformations. This is also the case of T-cells produced in thymus (an important component of the immune system) as a result of a complex sequence of proliferation, selection, death and maturation processes. Most models of the thymus are multi-compartmental and analyze the evolution of several populations. The corresponding mathematical models are based on interconnected differential equations, one for each population, which usually contain separate terms describing the growth, death and transfer of cells between populations. In mathematical biology there are several growth models, but the most used one is that of logistic type. This is also used by one of the most popular mathematical model of the thymus proposed by Mehr et al. in [3].

The variant of the Mehr's model we use in this paper describes the evolution of four populations of cells characterized by the absence or presence of some markers: double-negative population $(N)$, double-positive population $(D P)$ and single-positive populations $\left(M_{4}\right.$ and $\left.M_{8}\right)$. The mathematical model consists of a system of four differential equations (see Eq. (1)), which describes the evolution of the number of cells in each population.

$$
\left\{\begin{array}{l}
\dot{N}=r_{n} N\left(1-\frac{N}{K_{n}}\right)-d_{n} N-s_{n} N+b\left(1-\frac{N}{K_{n}}\right) \\
\dot{P}=r_{p} P\left(1-\frac{Z}{K}\right)-d_{p} P-\left(s_{4}+s_{8}\right) P+s_{n} N \\
\dot{M}_{4}=r_{4} M_{4}\left(1-\frac{Z}{K}\right)-d_{4} M_{4}-s_{04} M_{4}+s_{4} P \\
\dot{M}_{8}=r_{8} M_{8}\left(1-\frac{Z}{K}\right)-d_{8} M_{8}-s_{08} M_{4}+s_{8} P \\
Z=N+P+M_{4}+M_{8} .
\end{array}\right.
$$

Each equation involves several parameters associated with different processes (growth, death, transfer etc.). Thus, for the double-negative cells population the parameter $K_{n}$ represents the maximal individual capacity, $b$ denotes the number of progenitor cells that inflows daily into the thymus from the bone marrow, $r_{n}$ denotes the division or proliferation rate, $d_{n}$ denotes the death rate and $s_{n}$ denotes the rate of transfer between the $N$ and $D P$ populations. In the equations corresponding to the other three populations there is a logistic growth term which is controlled by the maximal carrying capacity of the thymus, denoted by $K$ and by the total number of T-cells in the thymus, denoted by $Z$. For $D P, M_{4}$ and $M_{8}$ populations the proliferation rates are denoted by $r_{p}, r_{4}$ and $r_{8}$, respectively. In a similar manner the death rates 
are denoted by $d_{p}, d_{4}$ and $d_{8}$. The transfer rates from the double positive cell population into the single-positive cell population are denoted with $s_{4}$ and $s_{8}$. The number of mature cells from populations $M_{4}$ and $M_{8}$ which are released in the blood are controlled by the transfer rates $s_{04}, s_{08}$, respectively. The Mehr's model is appropriate in describing the growth stage of the thymus until it reaches the stationary state. Obtaining information about the stability of the stationary state is an important aspect. A first stability analysis of the total population $Z$ is presented in [3]. However this analysis is based on several simplifying assumptions or particular cases. One of such simplifying assumption, which is valid in the early stage of the growth, is that $1-\frac{Z}{K} \approx 1$. In this case, the system (1) becomes a linear one. Other simplifications which allow the explicit computations of the fixed points of the right hand side of Eq. (1) and the derivation of stability conditions are based on apriori restrictions of parameters, e.g. equal rates for different populations, rates equal to zero etc. Experimental quantitative results suggest that such assumptions cannot always be made apriori.

Therefore, the aim of this paper is to analyze the stability of the stationary solutions of (1) based only on hypotheses which are in line with the experimental data. Moreover we analyze if the theoretically derived sufficient conditions for stability can be exploited in estimating numerical parameters which ensure the stability.

The approach is different than that in [3] also because we do not analyze only the stability of the global population but the stability of each population. This could be of practical interest as the small ratio of $M_{4}$ and $M_{8}$ populations in the total population makes difficult to infer knowledge on the behavior of these populations starting from results referring to the global population.

The paper is structured as follows. The second section presents the stablity results obtained under the assumption that the total population can be expressed, by linear regression, as a function of the dominant populations $(N$ and $P$ ). Section III presents the usage of the results derived in Section II in the context of constructing a model describing the evolution of a normal thymus starting from real experimental data. The last section concludes the paper.

\section{Stability analysis}

The direct computation of the stationary solutions of the system (1) is difficult, mainly because of the presence of the joint term $Z$ corresponding to the total number of cells in all populations. The solution proposed in [3] and [7] 
to simplify this computation is to consider the ratio $Z / K$ negligeable. This approximation is valid in the early stage of the evolution when the number of cells in each population is very small, but it is no more valid as the populations approach the stationary state. On the other hand, the double-negative and double-positive cells populations are dominant in $Z$, therefore $Z$ could be expressed as a function of $N$ and $P$, by neglecting the contributions of populations $M_{4}$ and $M_{8}$. Preliminary analysis based on experimental data suggested that there exists a linear dependence between $Z$ and $P$ and between $Z$ and $(N, P)$ (see Figures 1 and 2). Therefore the strategy we use to make the stability analysis easier is to replace $Z$ with $\alpha_{N} \cdot N+\alpha_{P} \cdot P+\beta$.

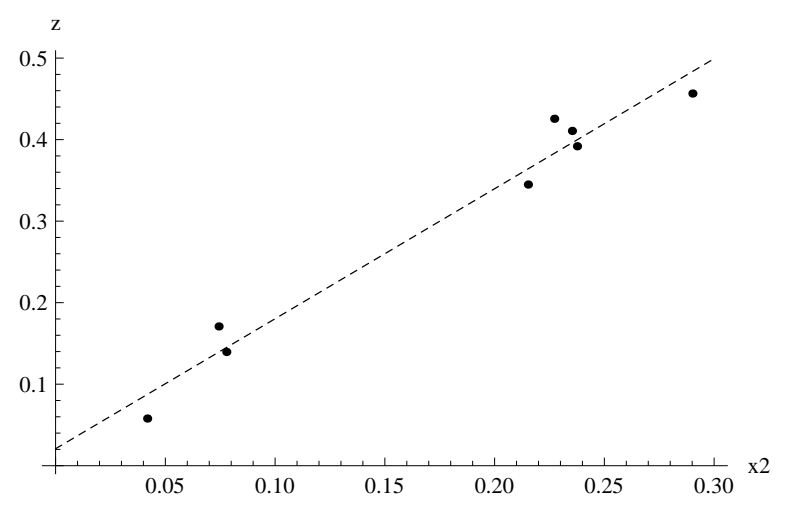

Figure 1: Regression line expressing the relationship between $x_{2}$ and $z: z=$ $1.5948 \cdot x_{2}+0.0208, R^{2}=0.969$

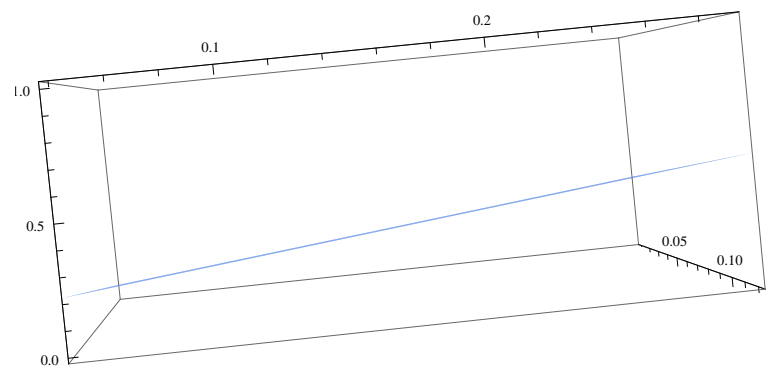

Figure 2: Regression plane expressing the relationship between $\left(x_{1}, x_{2}\right)$ and $z: z=1.4570 \cdot x_{1}+1.0516 \cdot x_{2}+0.0041, R^{2}=0.997$

In order to simplify the computation of the fixed points of (1) let us introduce the following substitutions (similar with those used in [3]): 


$$
\begin{aligned}
& a_{1}=r_{n}-d_{n}-s_{n}-\frac{b}{K_{n}} \\
& a_{2}=r_{p}-d_{p}-s_{4}-s_{8} \\
& a_{3}=r_{4}-d_{4}-s_{o 4} \\
& a_{4}=r_{8}-d_{8}-s_{o 8}
\end{aligned}
$$

and

$$
\begin{aligned}
x_{1} & =\frac{N}{K}, \quad x_{2}=\frac{P}{K}, \quad x_{3}=\frac{M_{4}}{K}, \quad x_{4}=\frac{M_{8}}{K} \\
z & =\frac{Z}{K}, \quad k=\frac{K}{K_{n}}, \quad \tilde{b}=\frac{b}{K} .
\end{aligned}
$$

With these notations the system (1) becomes:

$$
\begin{aligned}
& \dot{x}_{1}=a_{1} x_{1}-r_{n} k x_{1}^{2}+\tilde{b} \\
& \dot{x}_{2}=a_{2} x_{2}-r_{p} x_{2} z+s_{n} x_{1} \\
& \dot{x}_{3}=a_{3} x_{3}-r_{4} x_{3} z+s_{4} x_{2} \\
& \dot{x}_{4}=a_{4} x_{4}-r_{8} x_{4} z+s_{8} x_{2} .
\end{aligned}
$$

Since the presence of the joint term, $z=x_{1}+x_{2}+x_{3}+x_{4}$, makes the computation of the fixed points difficult we use the above mentioned idea of replacing $z$ with a linear combination of $x_{1}$ and $x_{2}$. We shall analyze two cases. In the first case, $z$ is expressed only with respect to the dominant component, i.e. $z=\alpha x_{2}+\beta$. In the second case, $z$ will be replaced with $z=\alpha_{1} x_{1}+\alpha_{2} x_{2}+\beta$.

\subsection{Case 1: $z=\alpha x_{2}+\beta$}

In this case (3) becomes:

$$
\begin{aligned}
& \dot{x}_{1}=a_{1} x_{1}-r_{n} k x_{1}^{2}+\tilde{b} \\
& \dot{x}_{2}=a_{2} x_{2}-r_{p} x_{2}\left(\alpha x_{2}+\beta\right)+s_{n} x_{1} \\
& \dot{x}_{3}=a_{3} x_{3}-r_{4} x_{3}\left(\alpha x_{2}+\beta\right)+s_{4} x_{2} \\
& \dot{x}_{4}=a_{4} x_{4}-r_{8} x_{4}\left(\alpha x_{2}+\beta\right)+s_{8} x_{2}
\end{aligned}
$$

We will consider only the stationary solution with positive components to be practically relevant, because the solutions of the differential system (4) represents the population of the thymus (which can't be negative). The stationary solution, $\left(x_{1}^{*}, x_{2}^{*}, x_{3}^{*}\right.$ and $\left.x_{4}^{*}\right)$, can be computed leading to: 


$$
\begin{aligned}
x_{1}^{*} & =\frac{a_{1}+\sqrt{a_{1}^{2}+4 \tilde{b} r_{n} k}}{2 r_{n} k}, \\
x_{2}^{*} & =\frac{a_{2}-\beta r_{p}+\sqrt{\left(a_{2}-\beta r_{p}\right)^{2}+4 \alpha r_{p} s_{n} x_{1}^{*}}}{2 \alpha r_{p}}, \\
x_{3}^{*} & =\frac{s_{4} x_{2}^{*}}{\alpha r_{4} x_{2}^{*}+\beta r_{4}-a_{3}}, \\
x_{4}^{*} & =\frac{s_{8} x_{2}^{*}}{\alpha r_{8} x_{2}^{*}+\beta r_{8}-a_{4}} .
\end{aligned}
$$

In order to analyze the stability properties of this stationary solution we construct the corresponding Jacobian matrix, $J$ :

$$
J=\left(\begin{array}{cccc}
j_{11} & 0 & 0 & 0 \\
j_{21} & j_{22} & 0 & 0 \\
0 & j_{32} & j_{33} & 0 \\
0 & j_{42} & 0 & j_{44}
\end{array}\right)
$$

where

$$
\begin{aligned}
& j_{11}=a_{1}-2 r_{n} k x_{1}^{*}, \\
& j_{21}=s_{n}, \\
& j_{22}=a_{2}-\beta r_{p}-2 \alpha r_{p} x_{2}^{*}, \\
& j_{32}=s_{4}-\alpha r_{4} x_{3}^{*}, \\
& j_{33}=a_{3}-r_{4}\left(\alpha x_{2}^{*}+\beta\right), \\
& j_{42}=s_{8}-\alpha r_{8} x_{4}^{*}, \\
& j_{44}=a_{4}-r_{8}\left(\alpha x_{2}^{*}+\beta\right) .
\end{aligned}
$$

Since the Jacobian matrix is triangular, the eigenvalues are the elements from the main diagonal $\left(j_{11}, j_{22}, j_{33}, j_{44}\right)$ :

$$
\begin{aligned}
& \lambda_{1}=-\sqrt{a_{1}^{2}+4 \tilde{b} r_{n} k}, \\
& \lambda_{2}=-\sqrt{\left(a_{2}-\beta r_{p}\right)^{2}+4 \alpha r_{p} s_{n} x_{1}^{*}}, \\
& \lambda_{3}=a_{3}-\beta r_{4}-\alpha r_{4} x_{2}^{*}, \\
& \lambda_{4}=a_{4}-\beta r_{8}-\alpha r_{8} x_{2}^{*} .
\end{aligned}
$$


From (6) it is easy to see that $\lambda_{1}$ and $\lambda_{2}$ are negative, which means that the stationary solutions coressponding to the first two populations $\left(x_{1}^{*}\right.$ and $\left.x_{2}^{*}\right)$ are stable. For values of the parameters tipically inferred from experimental data $\lambda_{1}$ and $\lambda_{2}$ are even strictly negative, thus $x_{1}^{*}$ and $x_{2}^{*}$ are asymptotically stable.

On the other hand, it is not obvious that $\lambda_{3}$ and $\lambda_{4}$ are always negative. Using (2) and (6) it follows that sufficient conditions to ensure the stability of the stationary solutions $x_{3}^{*}$ and $x_{4}^{*}$ are:

$$
\begin{aligned}
& \frac{d_{4}+s_{o 4}}{r_{4}}>1-\left(\alpha x_{2}^{*}+\beta\right), \\
& \frac{d_{8}+s_{o 8}}{r_{8}}>1-\left(\alpha x_{2}^{*}+\beta\right) .
\end{aligned}
$$

\section{$2.2 \quad$ Case 2: $z=\alpha_{1} x_{1}+\alpha_{2} x_{2}+\beta$}

Since the numerical regression suggests that this second regression model has a higher correlation coefficient $\left(R^{2}=0.969\right.$ in the first case and $R^{2}=0.997$ in the second case), it seems natural to analyze this case also.

Similar to the previous case we consider only the stationary solution with positive components to be relevant. For the second regression model the system (3) becomes:

$$
\begin{aligned}
& \dot{x}_{1}=a_{1} x_{1}-r_{n} k x_{1}^{2}+\tilde{b} \\
& \dot{x}_{2}=a_{2} x_{2}-r_{p} x_{2}\left(\alpha_{1} x_{1}+\alpha_{2} x_{2}+\beta\right)+s_{n} x_{1} \\
& \dot{x}_{3}=a_{3} x_{3}-r_{4} x_{3}\left(\alpha_{1} x_{1}+\alpha_{2} x_{2}+\beta\right)+s_{4} x_{2} \\
& \dot{x}_{4}=a_{4} x_{4}-r_{8} x_{4}\left(\alpha_{1} x_{1}+\alpha_{2} x_{2}+\beta\right)+s_{8} x_{2}
\end{aligned}
$$

and the corresponding stationary solution is:

$$
\begin{aligned}
x_{1}^{*}= & \frac{a_{1}+\sqrt{a_{1}^{2}+4 \tilde{b} r_{n} k}}{2 r_{n} k} \\
x_{2}^{*}= & \frac{\left(a_{2}-\beta r_{p}-\alpha_{1} r_{p} x_{1}^{*}\right)}{2 \alpha_{2} r_{p}}+ \\
& +\frac{\sqrt{\left(a_{2}-\beta r_{p}-\alpha_{1} r_{p} x_{1}^{*}\right)^{2}+4 \alpha_{1} r_{p} s_{n} x_{1}^{*}}}{2 \alpha_{2} r_{p}} \\
x_{3}^{*}= & \frac{s_{4} x_{2}^{*}}{\alpha_{1} r_{4} x_{1}^{*}+\alpha_{2} r_{4} x_{2}^{*}+\beta r_{4}-a_{3}} \\
x_{4}^{*}= & \frac{s_{8} x_{2}^{*}}{\alpha_{1} r_{8} x_{1}^{*}+\alpha_{2} r_{8} x_{2}^{*}+\beta r_{8}-a_{4}}
\end{aligned}
$$


The Jacobian matrix has again a triangular form:

$$
J=\left(\begin{array}{cccc}
j_{11} & 0 & 0 & 0 \\
j_{21} & j_{22} & 0 & 0 \\
j_{31} & j_{32} & j_{33} & 0 \\
j_{41} & j_{42} & 0 & j_{44}
\end{array}\right)
$$

where

$$
\begin{aligned}
& j_{11}=a_{1}-2 r_{n} k x_{1}^{*}, \\
& j_{21}=s_{n}-\alpha_{1} r_{p} x_{2}^{*} \\
& j_{22}=a_{2}-\alpha_{1} r_{p} x_{1}^{*}-2 \alpha_{2} r_{p} x_{2}^{*}-\beta r_{p}, \\
& j_{31}=-\alpha_{1} r_{4} x_{3}^{*} \\
& j_{32}=s_{4}-\alpha_{2} r_{4} x_{3}^{*} \\
& j_{33}=a_{3}-r_{4}\left(\alpha_{1} x_{1}^{*}+\alpha_{2} x_{2}^{*}+\beta\right), \\
& j_{41}=-\alpha_{1} r_{8} x_{4}^{*} \\
& j_{42}=s_{8}-\alpha_{2} r_{8} x_{4}^{*} \\
& j_{44}=a_{4}-r_{8}\left(\alpha_{1} x_{1}^{*}+\alpha_{2} x_{2}^{*}+\beta\right) .
\end{aligned}
$$

The eigenvalues of $J$ can be computed in a straightforward manner, because the Jacobian matrix has as well a triangular form, and so the eigenvalues are the elements from the main diagonal $\left(j_{11}, j_{22}, j_{33}, j_{44}\right)::$

$$
\begin{aligned}
\lambda_{1} & =-\sqrt{a_{1}^{2}+4 \tilde{b} r_{n} k}, \\
\lambda_{2} & =-\sqrt{\left(a_{2}-\beta r_{p}-\alpha_{1} r_{p} x_{1}^{*}\right)^{2}+4 \alpha_{1} r_{p} s_{n} x_{1}^{*}}, \\
\lambda_{3} & =a_{3}-r_{4}\left(\alpha_{1} x_{1}^{*}+\alpha_{2} x_{2}^{*}+\beta\right), \\
\lambda_{4} & =a_{4}-r_{8}\left(\alpha_{1} x_{1}^{*}+\alpha_{2} x_{2}^{*}+\beta\right) .
\end{aligned}
$$

As in the previous case, $\lambda_{1}$ and $\lambda_{2}$ are obviously negative, thus the stationary solutions $x_{1}^{*}$ and $x_{2}^{*}$ are stable. On the other hand the sufficient conditions ensuring the stability of $x_{3}^{*}$ and $x_{4}^{*}$ are:

$$
\begin{aligned}
& \frac{d_{4}+s_{o 4}}{r_{4}}>1-\left(\alpha_{1} x_{1}^{*}+\alpha_{2} x_{2}^{*}+\beta\right), \\
& \frac{d_{8}+s_{o 8}}{r_{8}}>1-\left(\alpha_{1} x_{1}^{*}+\alpha_{2} x_{2}^{*}+\beta\right) .
\end{aligned}
$$


These conditions are very similar to those in (7) and provides constraints on the rates describing the evolution of $M_{4}$ and $M_{8}$ populations. These constraints will be used in the following section in order to estimate values for the rates which ensures that the stationary states of all populations are asymptotically stable.

\section{A case study on experimental data for mouse thymus}

In an excellent review [1] of recent developments in parameter estimation and structure identification of biological systems, Chou and Voit described the main phases in constructing a mathematical model. Data selection, specification of assumptions and simplifications and parameter estimation are some of the nine phases mentioned in [1].

\subsection{Data selection}

In order to analyze the practical impact of the stability results obtained in the previous section we conducted a case study based on a set of experimental data concerning T-cells populations evolving in mouse thymus during the first two months of life which correspond to the growth period. More specifically, the data were obtained by counting, using specific techniques, the number of double-negative, double-positive, $M_{4}$ and $M_{8}$ cells at some specific time points, i.e. 5, 10, 15, 20, 25, 30, 35, 40 days. For each time point, values recorded for at least three mice were provided. Based on the raw data, the average value for each time point was computed and the resulting set of values were used in simulations.

Using the substitutions and notations introduced in the previous section (see Eq. (3)), the experimental data can be specified as a set $\left\{\left(t_{j}, \bar{x}_{1 j}, \bar{x}_{2 j}, \bar{x}_{3 j}, \bar{x}_{4 j}\right)\right.$; $j=\overline{1, N}\}$ where $\bar{x}_{i j}$ denotes the average value of experimental data for population $i$ recorded at time $j$.

\subsection{Assumptions and simplifications}

The main assumption in the numerical simulation of the mathematical model (3) is that used also in the theoretical stability analysis, namely the fact that $z$ depends linearly on $x_{1}$ and $x_{2}$, i.e. $z=\alpha_{1} \cdot x_{1}+\alpha_{2} \cdot x_{2}+\beta$. The values of $\alpha_{1}, \alpha_{2}$ and $\beta$ were estimated using linear regression applied on the values obtained by transforming the averaged experimental data according to Eq. (3) for $K=450 \cdot 10^{6}$. This value of $K$ is related to the total carrying capacity 
of the thymus and was obtained by a preliminary estimation based on the experimental data. It is in accordance with values reported in the literature $[5],[2]$.

\subsection{Parameter estimation}

There are 16 parameters of the model described by Eq. (1) which should be estimated. These parameters represent [3]: cells proliferation rates $\left(r_{n}, r_{p}\right.$, $\left.r_{4}, r_{8}\right)$, cells death rates $\left(d_{n}, d_{p}, d_{4}, d_{8}\right)$, transfer rates between populations $\left(s_{n}, s_{4}, s_{8}, s_{04}\right.$ and $\left.s_{08}\right)$, seeding rate of $\mathrm{T}$ cells progenitors from bone marrow (b) and an upper bound for the number of double negative cells $\left(K_{n}\right)$. For all these parameters there are known some ranges of values which are biologically plausible (see for instance [3], [4]). These ranges define the parameter space which should be searched in order to estimate values for the parameters which ensures that the model fits to the data. The search ranges we used in the numerical estimation of parameters include the ranges provided in the literature. More specifically, we used the range $[0,2]$ for the proliferation rates $\left(r_{n}, r_{p}, r_{4}\right.$ and $\left.r_{8}\right)$ and the range $[0,1]$ for all the other parameters, except for $b$ and $K_{n}$ for each we used $\left[10^{2}, 10^{3}\right]$ and $\left[30 \cdot 10^{6}, 200 \cdot 10^{6}\right]$.

The task of estimating the parameters can be formulated as an optimization problem aiming to minimize the distance between experimental data and model predictions. A typical distance is the mean squared error (MSE) which in the case of the model (3) involving four differential equations can be described as:

$$
\operatorname{MSE}(\sigma)=\frac{1}{4 N} \sum_{i=1}^{4} \sum_{j=1}^{N}\left(x_{i}\left(\sigma ; t_{j}\right)-\bar{x}_{i j}\right)^{2}
$$

where $\sigma=\left(r_{n}, r_{p}, r_{4}, r_{8}, d_{n}, d_{p}, d_{4}, d_{8}, s_{n}, s_{4}, s_{8}, s_{o 4}, s_{o 8}, b, K_{n}\right)$ denotes the set of parameters and $x_{i}(\sigma ; t)$ is a numerically estimated solution for the given set of parameters.

A particularity of our approach is that we are looking for parameter values which ensures the asymptotic stability of the stationary states $x_{3}^{*}$ and $x_{4}^{*}$. Based on the stability results obtained in the previous section it follows that the parameters values satisfying Eq. (12) ensures the desired stability. Therefore the optimization problem to be solved becomes a constrained optimization problem with constraints given by Eq. (12). One of the simplest approaches in dealing with constrained optimization problems is the penalty method which includes the constraints into the objective function. In our case the objective function becomes: 


$$
\begin{aligned}
M S E_{c}(\sigma)= & c_{1} \cdot M S E(\sigma) \\
& +c_{2} \cdot \sum_{k \in\{4,8\}} \max \left(0,1-z^{*}(\sigma)-\frac{d_{k}+s_{o k}}{r_{k}}\right)
\end{aligned}
$$

where $z^{*}(\sigma)=\alpha_{1} \cdot x_{1}^{*}(\sigma)+\alpha_{2} \cdot x_{2}^{*}(\sigma)+\beta, x_{i}^{*}(\sigma)$ denotes the corresponding stationary solution obtained for the values of parameters specified in $\sigma$ and $c_{1}, c_{2} \in[0,1]$ are the penalty coefficients satisfying $c_{1}+c_{2}=1$. In the numerical simulation we used $c_{1}=c_{2}=0.5$.

Since the differential equations involved in the model cannot be solved analytically, there is no an explicit form of the dependence between the objective function $M S E_{c}$ and the parameters to be estimated. In most cases it is difficult to know the structure of the objective function landscape, therefore gradient-based optimization methods can get trapped in local minima. Instead, population-based stochastic methods, as evolutionary algorithms, proved to be effective in solving parameter estimation tasks [1]. These methods use a population of solution candidates, ensure the exploration of the search space by using specific operators to create new potential solutions and guide the search toward the optimum by specific selection rules. From the large family of population-based stochastic methods we selected an adaptive differential evolution algorithm called JADE which was introduced in [8]. The main reasons of selecting this method are: its flexibility, robustness to the choice of control parameters and the large number of successful applications in various optimization problems [6].

The stochastic character of the optimization method (induced both by the random values assigned to the initial population and by the random character of the search operators) is usually a drawback. In the context of the parameter estimation problem we exploited this characteristic by producing, through several independent runs of the estimation process, a sample of parameter values. This values are used to construct confidence intervals for all those sixteen parameters of the mathematical model. Such an approach is advantageous, since it is unlikely that an unique set of parameters provide enough information about the model particularities.

\subsection{Implementation and results}

In order to estimate confidence intervals for the parameters, we run the optimization method for $S=10$ times starting from different initial populations of parameters. At each iteration of the JADE algorithm, the quality of each element of the population (which is a vector containing current estimations of the parameters) is computed by numerically solving both the differential equations system and the algebraic system producing the stationary solution and by evaluating the objective function $M S E_{c}$. The parameter estimation 
algorithm was implemented in Mathematica 7.0, therefore both the differential and the algebraic systems were solved using the numerical procedures implemented in this software package.

The results presented in this paper were obtained by running the JADE algorithm for a population of 50 elements for 500 iterations. The confidence intervals obtained from the parameter values provided in $S$ independent runs are presented in Table 1 while the stationary solutions and the corresponding values of the eigenvalues $\lambda_{3}$ and $\lambda_{4}$ are presented in Table 2. Since all eigenvalues are negative it follows that the proposed constrained estimation procedure leads to parameters which ensures the asymptotic stability of the stationary solutions corresponding to all four populations of cells. Moreover it is easy to check using the limits of the confidence intervals that since $\left(d_{4}^{(\min )}+s_{o 4}^{(\min )}\right) / r_{4}^{(\max )}>1$ it follows that for any value of the parameters in the confidence intervals the $\lambda_{3}$ eigenvalue is strictly negative, thus $x_{3}^{*}$ is asymptotically stable. In the case of $x_{4}^{*}$ even if $\left(d_{8}^{(\min )}+s_{o 8}^{(\min )}\right) / r_{8}^{(\max )}<1$ the corresponding eigenvalue is negative for values of $z^{*}$ larger than 0.204259 (which is obviously satisfied by all stationary states having values close to those reported in Table 2).

\begin{tabular}{cccc}
\hline \hline$r_{n}$ & $r_{p}$ & $r_{4}$ & $r_{8}$ \\
$(0.859,1.041)$ & $(0.241,0.349)$ & $(0.247,1.069)$ & $(0.451,1.033)$ \\
\hline$d_{n}$ & $d_{p}$ & $d_{4}$ & $d_{8}$ \\
$(0.066,0.285)$ & $(0.034,0.152)$ & $(0.718,0.908)$ & $(0.575,0.836)$ \\
\hline$s_{n}$ & $s_{4}$ & $s_{8}$ & \\
$(0.374,0.633)$ & $(0.148,0.207)$ & $(0.067,0.135)$ & \\
\hline$s_{o 4}$ & $s_{o 8}$ & $b$ & $K_{n} / 10^{6}$ \\
$(0.727,0.897)$ & $(0.247,0.796)$ & $(153,376)$ & $(154,184)$ \\
\hline \hline
\end{tabular}

Table 1: Estimated confidence intervals for the parameters involved in the mathematical model (1).

The behavior of $x_{1}(t), x_{2}(t), x_{3}(t)$ and $x_{4}(t)$ for a set of estimated parameters is illustrated in Figures 3 and 4 . As these plots suggest, the approximation quality is worse in the case of $x_{3}$ and $x_{4}$. This can be explained by the fact that the differences in the data corresponding to different cells populations lead to different contributions of the populations to the $M S E$ part of the objective function. Therefore the selection pressure stimulates more the quality of $x_{1}$ and $x_{2}$ approximation and less the quality of $x_{3}$ and $x_{4}$ approximation. In order to deal with this issue the parameter estimation process shoul be split in several processes corresponding to the different cells types but this 


\begin{tabular}{ccccccc}
\hline run & $x_{1}^{*}$ & $x_{2}^{*}$ & $x_{3}^{*}$ & $x_{4}^{*}$ & $\lambda_{3}$ & $\lambda_{4}$ \\
\hline 1 & 0.10248 & 0.25156 & 0.04285 & 0.03098 & -1.50 & -1.47 \\
2 & 0.10889 & 0.26114 & 0.03464 & 0.03257 & -1.13 & -0.93 \\
3 & 0.11064 & 0.26319 & 0.03777 & 0.03462 & -1.34 & -0.67 \\
4 & 0.11156 & 0.26130 & 0.03736 & 0.03241 & -1.27 & -0.80 \\
5 & 0.11042 & 0.26813 & 0.03097 & 0.03360 & -0.86 & -1.00 \\
6 & 0.09927 & 0.25941 & 0.03601 & 0.03258 & -1.35 & -1.05 \\
7 & 0.10283 & 0.26433 & 0.03728 & 0.03282 & -1.06 & -0.81 \\
8 & 0.11016 & 0.25786 & 0.03806 & 0.03307 & -1.36 & -0.84 \\
9 & 0.10816 & 0.26111 & 0.03498 & 0.00624 & -1.17 & -0.02 \\
10 & 0.09930 & 0.26107 & 0.03568 & 0.03400 & -1.46 & -0.42 \\
\hline
\end{tabular}

Table 2: Set of stationary solutions obtained in the $S=10$ runs of the parameter estimation algorithm

aspect is out of the scope of the current paper.

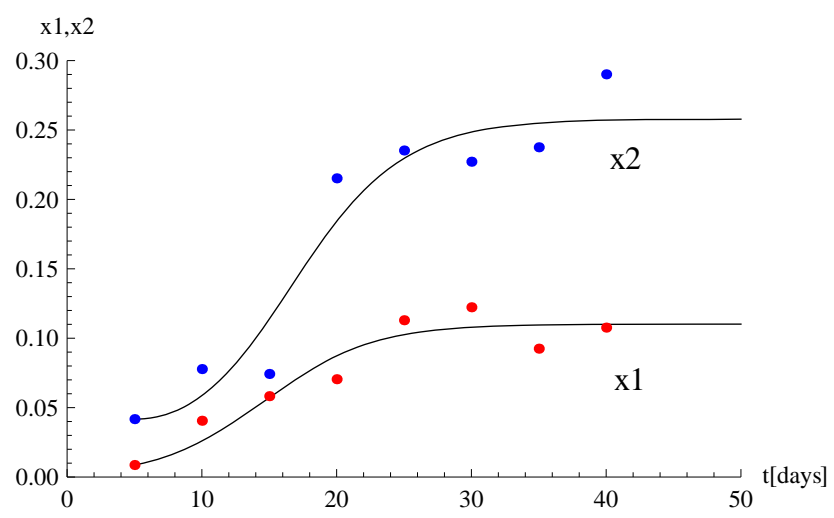

Figure 3: The evolution of $x_{1}(t)$ and $x_{2}(t)$ for the parameters estimated in run $8(M S E=0.0002011)$. Red points corresponds to averaged experimental data associated to $x_{1}$ while blue points are associated to $x_{2}$

\section{Conclusions}

The stability analysis conducted in this paper is based on the fact that the original Mehr's model can be simplified by using the remark that the total 


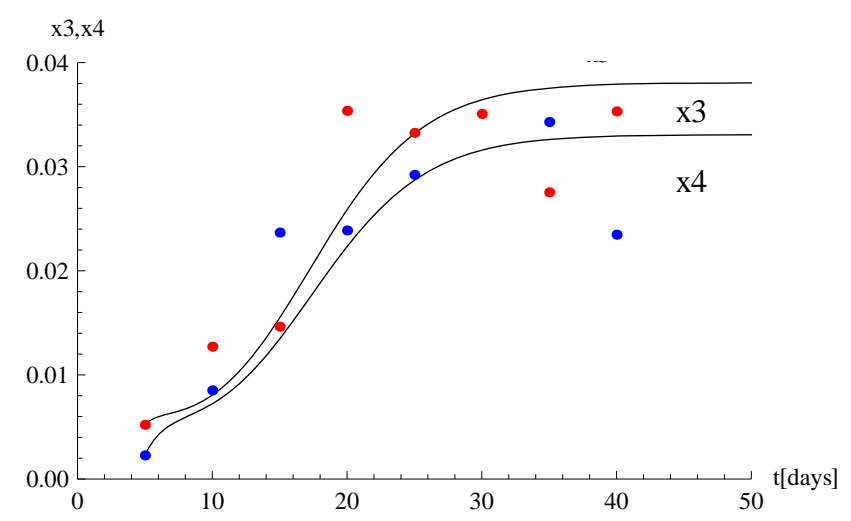

Figure 4: The evolution of $x_{3}(t)$ and $x_{4}(t)$ for the parameters estimated in run $8(M S E=0.0002011)$. Red points corresponds to averaged experimental data associated to $x_{3}$ while blue points are associated to $x_{4}$

number of cells in all four populations can be expressed using only the dominant populations. Linear regression based on experimental data allowed us to find an explicit dependence between $Z$ and $(P, N)$. Using this simplification, which is valid not only in the early stage of the evolution, the stationary solutions can be computed explicitely and sufficient conditions for stability can be derived by computing the eignevalues of the corresponding Jacobian matrix. For the first two populations $(N$ and $P$ ) the corresponding eigenvalues are strictly negative, ensuring the asymptotic stability of the stationary solutions, as it has been also proved, under different assumptions in [3] and [7]. On the other hand, for the last two populations the fact that the eigenvalues are negative is not so obvious. In this case we used the stability condition as a constraint in the optimization process which allows to estimate the model parameters. In this way we obtained confidence intervals for the parameters such that any set of parameter values selected from these intervals ensures the asymptotic stability of the stationary state.

\section{Acknowledgment}

The authors thanks dr. Felix Mic for providing the experimental data. This work was partially supported by the PN-II-ID-PCE-2011-3-0571 grant "Modeling and simulation of the dynamics of thymocyte populations and cells of the thymus medulla under normal and pathological situations", 2012-2014 and by the strategic grant POSDRU/CPP107/DMI1.5/S/78421, Project ID 
78421 (2010), co-financed by the European Social Fund Investing in People, within the Sectoral Operational Programme Human Resources Development 2007-2013.

\section{References}

[1] I.C. Chou and E.O. Voit, Recent developments in parameter estimation and structure identification of biochemical and genomic systems, Mathematical Biosciences, 219, (2009), 57-83

[2] J.S. Hale, T.E. Boursalian, G.L. Turk, and P.J. Fink, Thymic output in aged mice, PNAS, 103 (22), (2006), 84478452

[3] R. Mehr, A. Globerson, and A. Perelson, Modeling Positive and Negative Selection and Differentiation Processes in the Thymus, J. Theor. Biol., 175, (1995), 103-126

[4] R. Mehr, A. Perelson, M. Fridkis-Hareli, and A. Globerson, Feedback regulation of $\mathrm{T}$ cell development: manifestations in aging, Mechanisms of Ageing and Development, 91, (1996), 195-210

[5] C.L. Ortman, K.A. Dittmar, P.L. Witte, and P.T. Le, Molecular characterization of the mouse involuted thymus: aberrations in expression of transcription regulators in thymocyte and epithelial compartments, International Immunology, 14 (7), (2002), 813-822

[6] P. Posik and V. Klema, JADE, an adaptive differential evolution algorithm, benchmarked on the BBOB noiseless testbed, Proceedings of GECCO 2012, 2012, 197-204

[7] H. Shi, W. Ma, and Z. Duan, Global asymptotic stability of a nonlinear timedelayed system of T cells in the thymus, Nonlinear Analysis, 71, (2009), 2699-2707

[8] J. Zhang and A.C. Sanderson, JADE: adaptive differential evolution with optional external archive, IEEE Trans. on Evolutionary Computation, 13 (5), (2009), 945-958

Lavinia Cristina Moatăr-Moleriu

Faculty of Mathematics and Computer Science, West University of Timişoara, 4 blvd. V. Pârvan

\& Department of Functional Sciences, Victor Babes, University of Medicine and Pharmacy, 2 Eftimie Murgu Sq.

Timişoara, Romania

E-mail: lavimoatar@yahoo.com

Received: 1.09.2013

Accepted: 10.12.2013 